\title{
Cancer Prevention and Control in Pakistan: Review of Cancer Epidemiology and Challenges
}

\author{
Uzma Shamsi ${ }^{*}$ \\ ${ }^{1}$ Department of Community Health Sciences, Aga Khan University, Karachi, Pakistan
}

\begin{abstract}
With current situation of increasing burden of cancer in Pakistan, this report reviews studies related to cancer burden and cancer prevention and control in Pakistan.

Electronic databases used were PubMed, Medline, EMBASE, the University of Adelaide library \& Aga Khan University (AKU) library database, to search for relevant articles on the topic of cancer prevalence and prevention in Pakistan published in English. We conducted a literature search of published epidemiological and clinical studies relating to this topic up to March 1, 2020. It showed that there is a dearth of epidemiological studies in cancer risk factors, prevention and control in our population. Most studies are hospital based with small sample sizes and underpowered.

Epidemiological integrated transdisciplinary research is needed to find the actual burden, etiology and feasible prevention strategies of cancer unique to our population with focus on low cost screening methods for early detection of cancers and premalignant lesions in our population. There is an urgent need for a centralized national cancer registry to have combined results of cancer registry from Pakistan Atomic Energy Commission, Karachi Caner Registry, Punjab Cancer Registry and cancer registries from the rest of Pakistan. To have the data of cancer incidence in Pakistan, policy is needed to make cancer incidence as notifiable disease and mandatory. Collaboration is also needed to work with diverse groups like the National Cancer Society of Pakistan (NCSP), National Cancer Control Plan, and Society of Medical Oncology Pakistan to draft and implement plans and strategies for national cancer control programs. It will help mobilize Ministry of Health and policy makers to address the alarming high incidence of different types of cancers in Pakistan.
\end{abstract}

Keywords: Cancer prevalence, cancer epidemiology, risk factors, cancer prevention and screening, palliative care, cancer registry, Pakistan.

\section{BACKGROUND}

The global burden of cancer is increasing and there was approximately 8.1 million new cases with 9.6 million deaths in 2018 [1]. According to an estimate the risk of developing cancer is one in 5 among men and one in 6 among women during their lifetime [2]. Globally, the most common cancers are cancers of lung, female breast, and colorectum. In Asia, it is observed that there has been one of the most rapid increases in breast cancer occurring over the last two decades [3]. With current situation of increasing burden of cancer in Pakistan, this report reviews studies related to cancer burden and cancer prevention and control in Pakistan.

\section{METHODS}

Electronic databases used were PubMed, Medline, EMBASE, the University of Adelaide library \& AKU library database, to search for relevant articles on the topic of cancer prevalence in Pakistan published in English. We conducted a literature search of published epidemiological and clinical studies relating to this topic up to March 1, 2020. Following key terms were used: cancer prevalence, cancer epidemiology, risk factors, cancer prevention and screening, palliative care, Punjab

\footnotetext{
*Corresponding Author: Uzma Shamsi, Department of Community Health Sciences, Aga Khan University, Karachi, Pakistan;

Email: uzma.shamsi@aku.edu

Received: February 23, 2020; Revised: April 21, 2020; Accepted: May 04, 2020

DOI: https://doi.org/10.37184/lnjpc.2707-3521.1.20
}

cancer registry, Karachi cancer registry combined with Pakistan. EndNote (X8.1) was used to create a reference database and finally 49 articles were included after excluding 45 articles.

\section{CANCER IN PAKISTAN}

In Pakistan, approximately 150, 000 new cases of cancer are diagnosed annually, with high mortality of 60\%-80\% [4]. Data from the Karachi Cancer Registry (KCR) reported that most common cancer was lung cancer among males, associated with cigarette smoking followed by carcinoma of the oral cavity, again associated with high tobacco smoking and smokeless tobacco [5] in spite of smoking ban in Pakistan since 2002 [6]. Breast cancer was the most common malignancy in females with a peak at the age of around 45 years. The second and the third most common malignancies amongst the females were of the oral cavity (observed in the second and third decades of life) and the ovary [7]. Like other lower and middle-income countries (LMICs), breast cancer incidence is rising rapidly in Pakistan too [8, 9]. Hospital based studies of breast cancer are constantly reporting breast cancer at a younger age in their 40s and with locally advanced disease among Pakistani women, imposing a bigger public health concern in future for Pakistan [10-12]. Results from a pathology laboratory based cancer registry for the period of 2010-2015 among people from all districts of Karachi showed that Karachi has the highest number of cancers of breast, head \& 
neck and esophagus in females and cancer of lip and oral cavity and larynx in males (associated with all forms of tobacco) compared to any of the Asian populations [13]. Stomach cancer has also been reported mostly in young Pakistani males [14].

\section{CANCER PREVALENCE RATES VARIATIONS}

A recent meta-analysis of 13 studies done in Pakistani population showed a significant variation in the reported prevalence rates of different cancers (breast cancer, oral cancer, prostate cancer, gastric cancer and colorectal cancer). The prevalence rates of these cancers differed in different studies according to the different places and populations [15]. Even commonest cancers in males and females also varied in different hospital-based data. Oral cancer varied from $2 \%$ to as high as $19 \%$ : prostate cancer ranged from $2.2 \%$ to as high as $8.8 \%$ with overall pooled prevalence of $5 \%$, breast cancer from $20-50 \%$ with pooled prevalence of $31 \%$ and prevalence of stomach cancer varied from $1.4 \%-22 \%$. Finally, the prevalence of colorectal cancer CRC ranged from as 4.3\%-7.1\%. According to the report by GLOBOCON 2012, lung cancer was the 3rd most common cancer in Pakistan while Pakistan Health Research Council (PHRC) 2016 has estimated it as the 10th most common cancer over the past one decade [16]. Cancer data from Lahore in 1984-2014 showed lymphomas and breast cancer as the most common neoplasm in males and females, respectively, and high incidence of head and neck, brain, and lung cancers, and leukemia among males, and ovarian, cervix, head and neck and lymphomas among females [17]. Another study by KCR showed that the incidence of cancer ovary, involved younger age group with a positive family history of ovarian cancer and advanced stage at presentation [18].

In another retrospective, observational study in the Shaukat Khanum Memorial Cancer Hospital and Research Centre (SKMCH \& RC), Lahore, during 18 years' time period (1994 to 2012) the most common malignancies among adults, were those of breast, lip and oral cavity, and liver and intrahepatic bile ducts [19].

The Pakistan Atomic Energy Commission Cancer Registry (PAECCR) database for the last 30 years (1984-2014) confirmed that head and neck and breast cancers in males and in females respectively were the most common cancers in Punjab province in Pakistan, highest in Asia. Moreover, the incidence of cancers of brain, prostate and non-Hodgkin's Lymphoma NHL among males and ovarian and cervix cancers among females were observed to be increasing rapidly [20]. According to it, head and neck cancer was $13.41 \%$, brain tumor was $10.90 \%$, and non-Hodgkin lymphoma $\mathrm{NHL}$ was $9.70 \%$ among males, whereas breast cancer was $45 \%$, ovary tumors was $6.6 \%$, and head and neck was $6.21 \%$ in females. Leukemia was reported as the most common cancer for the age group of 1-12 years [21]. PAECR report of 2015-2017 of more than 100,000 cancer patients in 18 hospitals of Pakistan again confirmed head and neck cancer and breast cancer as most common cancers. Median age of breast cancer (49 years) among Pakistani women is much younger age compared with other populations' median age at breast cancer diagnosis [22]. According to this report, oral cavity, lung and CRC among males and Breast, oral cavity and CRC among females were the major cancers.

The Shaukat Khanum Memorial Cancer Hospital \& Research Centre (SKMH\&RC) reviewed cancer among patients 0-19 years of age, from 2011 to 2012 and reported 669 new cases of common childhood malignancies like leukemia, myeloproliferative/dysplastic disorders, lymphoma, reticuloendothelial neoplasms, central nervous system CNS and miscellaneous intracranial/ intraspinal tumors and malignant bone tumors [23].

\section{RISK FACTORS}

Regarding the etiology of cancers, there is interaction of both genetic and environmental factors like poor lifestyle behaviors in the development of specific cancers in our population. There are changing trends in cancer incidence due to changing demographic and lifestyle factors as it is reported that there has been a reduction in incidence of esophageal and gastric cancers due to better diet and better SES in certain high-risk areas, whereas in countries like the United States, Australia, and France, the decreased in incidence is attributed to declines in cigarette smoking [24]. According to Institute of Nuclear Medicine and Oncology, Lahore, Pakistan, the main risk factors of head and neck cancer (18.74\%) recorded during $2004-2014$ were related to smoking, drinking and smokeless tobacco ("paan", "gutka"), age, low socioeconomic status, family history of cancer, occupation in chemical factories [25]. There are 16000 new cases annually of oral cancer, with strong relationship between oral cancer and "naswar" (powdered tobacco) usage in Pakistan [26]. A review of 11 years (20052015) concluded that infections associated with human papillomavirus was the greatest risk of carcinoma cervix and high mortality rate [27]. In twin cities of Pakistan, a study was conducted to screen cervical cancer patients for HPV infection in a small sample of 67 cervical cancer biopsies. It showed a strong association between HPV infection and cervical cancer and it is an evidence for the need of prophylactic HPV vaccines and PAP smear screening [28].

A genetic epidemiology study done on 370 patients reported PALB2 mutations in early-onset and hereditary breast/ovarian cancer cases and importance of genetic etiology of breast cancer [29]. There is an immense need of Pakistani breast/ovarian cancer cases genetic testing for BRCA1/2 mutations and genetic testing of high-risk Pakistani breast/ovarian cancer [30]. A study in 2002 reported $6.7 \%$ of BRCA1 or BRCA2 mutations among breast cancer cases, with higher proportion of premenopausal breast cancer [31]. 


\section{LACK OF AWARENESS ABOUT CANCER}

Research studies have shown that $30-50 \%$ cancer cases can be avoided by eliminating or reducing exposure to known lifestyle and environmental risk factors [32, 33]. Studies show that Pakistani people have little awareness about common cancers like breast, cervix, head and neck cancers, its risk factors, and consumption of substances causing cancer and unrelated causes of cancer like black magic and fate were attributed to cancer [34-36]. Measures are needed for the awareness about breast cancer [37] as increased awareness for recognition of symptoms for breast cancer detection and timely referrals by multiple healthcare providers, especially traditional healers and general practitioners in Pakistan, can decrease the delay in diagnosis [38]. Abandonment of treatment was also found high among patients with childhood malignancies in a study between November 2014 and May 2016, in spite of free treatment in a welfare hospital [39]. Delayed diagnosis of oral cancers was common, frequently due to patient's self-delay and the main reason was lack of awareness about oral cancer risk factors, symptoms, and whom to approach for treatment [40]. Timely referral can decrease the complications and mortality due to oral cancers which is preventable if use of cigarette, "paan", "gutka is banned.

\section{LEVELS OF SCREENING AND PREVENTION}

Up to one-third of cancers in the developing world are curable if recognized early [41]. Pakistan has no national breast cancer screening or awareness programs and it is an important reason that women present at a more advanced stage [42]. Delayed presentation of cancer has a significant economic impact, since it is far less expensive to treat patients with early-stage disease and success rates are significantly increased. To minimize the delay in diagnosis, it is important to implement an organized breast cancer screening program, at primary care level, through providing low-cost mammograms, with a target population of women especially high-risk women and try to outreach into the public sector at community level including both urban and rural areas.

Cervical cancer is the most commonly and easily diagnosed cancer in the developed countries due to effective screening program PAP screening which is lacking in Pakistan. However, the situation can be transformed to accelerated declines in cervical cancer like in high-income countries through implementing resource-dependent programs of Visual Inspection with Acetic Acid (VIA), PAP screening and HPV vaccination for cervical cancer screening and prevention [43].

A study in SKCMH to assess the burden of Ca cervix showed that current HPV vaccine could potentially prevent new cervical cancer cases [44]. Similarly, there exist many barriers to CRC screening at Primary Health Care PHC level in a resource constraint country like Pakistan, with poor health literacy.
A small study conducted among Karachi residents reported $2.6 \%$ of CRC screening in eligible individuals (50 years or older) but only $14.9 \%(n=58)$ of study participants showed interest for CRC screening. There was severe lack of awareness about the CRC due to many barriers like poor health literacy [45].

\section{PALLIATIVE CARE}

Given the higher morbidity and highest mortality, cancer is now a major public health burden and challenge. There is dearth of well-equipped cancer hospitals with only 20 cancer hospitals in Pakistan where approximately 320,000 of new cases of cancer are expected every year. Moreover, for advanced and terminal cancer care, there are no palliative care facilities or hospices [46]. A study done in Mayo hospital Lahore showed under treatment of cancer pain in Pakistan is alarming due to inadequate training of health care providers, patients beliefs, lack of availability of opioid medications, and socioeconomic factors [47]. In government hospitals it is observed that there is an overwhelming numbers of advanced cancer patients and an enormous need for opioids, with lack of medication even for agonal breathing of a dying child [48].

\section{ASSESSING HUMAN AND ECONOMIC BENEFITS OF CANCER PREVENTION}

The financial burden of cancer care is also huge and overwhelming due to multiple factors [49]. It is time to focus on cost effective cancer prevention and to have data about the relative costs and outcomes of cancer control and prevention activities as healthcare resources are limited. An increase in the proportion of cancers of breast, colorectum, mouth detected clinically at an early stage in the absence of screening is commonly referred to as downstaging which is cost effective and beneficial in our setting.

\section{CANCER SURVEILLANCE, CANCER REGISTRY AND OTHER DATA SOURCES OF CANCER}

There is no data of cancer incidence in Pakistan till date as the notification of cancer is not yet mandatory. The incidence, prevalence, survival and mortality data of cancer available is mostly from hospital and laboratory. For the past 71 years there is no national centralized cancer registry in Pakistan to effectively unify and coordinate data from across the country. Few population-based studies have been conducted so far and most studies are done from discreet hospital based data sources [34].

KCR which was the first population-based registry of Pakistan, by the Government of Sindh in collaboration with the International Agency for Research on Cancer (IARC), WHO in 1995 and covered Southern Karachi in Pakistan. It contributed important publications of population based studies on different types of cancer. Punjab Cancer Registry (PCR) was set up in February 
2005 and is being run and sponsored by Shaukat Khanum Memorial Cancer Hospital \& Research Center, a private cancer treatment hospital. It has been successful in collecting data on cancer cases (though still 60\%) from various centers of the Province. The underreporting is due to a lack of priority to cancer disease surveillance, hesitation for data sharing by some hospitals, lack of funds and infrastructure. Before these registries, Cancer Registry of the Jinnah Postgraduate Medical Centre (JPMC) and the Pakistan Medical and Research Council (PMRC) was the first and closest representative of the data of Karachi, as it was the only cancer institute in Karachi.

Hospital-based results obtained from various oncology hospital and departments can be considered as an effective way forward in assessing some cancer burden in the region but the results can't be extrapolated to the whole population of the country and devise appropriate screening and therapeutic strategies according to cancer burden in the country [48].

\section{NEED FOR CANCER PREVENTION RESEARCH AND EDUCATION}

Epidemiological integrated transdisciplinary research is needed to find the actual burden, etiology and feasible prevention strategies of cancer unique to our population. There is need to conduct more research on cancer prevention identify risk factors, low cost screening methods and early detection of cancers and premalignant lesions in our population. With research using local data, we can expect improvement in cancer mortality. Evidence based data is needed in cancer epidemiology in collaboration with non-government organizations NGOs and Ministry of Health for implementation of cancer screening programs at PHC level. It will help mobilize Ministry of Health and policy makers to address the alarming high incidence of different types of cancers in Pakistan. There is also a greater need to emphasize cancer prevention education at both in under-graduation and graduation level, with introduction of cancer epidemiology and cancer prevention courses.

\section{USE OF TELEMEDICINE IN CANCER PREVENTION}

There is immense scope to use telecommunications technology to provide health care services to patients and in both primary and secondary cancer prevention like smoking prevention, nutritional counselling, exercise education, genetic counselling, psychosocial support, screening for breast cancer, cervical cancer, oral cancer, and CRC. It is cost-effective, accessible and clinically efficient with better compliance and satisfaction among patients especially those with limited access to health care.

\section{INTEGRATING CANCER PREVENTION AT PRIMARY HEALTH CARE LEVEL}

At PHC level, there is an urgent need for intensive and comprehensive education campaigns about cancer awareness, its risk factors, screening and prevention of common cancers. It is important to include health care providers, clinicians, nurses, community health workers, and lady health visitors in these educations. There is immense potential for public health professionals at primary health care (PHC) level to play their role in cancer control. It is important to promote awareness about oral, colorectal cancer and breast cancer among GCPs, and nurses at PHC by conducting training program for detection of precancerous and early-stage cancers and timely referral to oncologist/surgeon without delay and encouraging cancer screening activities especially for people with high risk profile. GCPs should be encouraged to do clinical breast examination (CBE) for breast cancer and oral examination regularly for any precancerous lesions. Lady health workers' training for breast cancer awareness education, breast selfexamination (BSE), clinical breast examination (CBE) and through them educate women about breast selfexamination is also cost-effective way to control cancer in our resource constraint population. Collaboration is also needed to work with diverse groups like the National Cancer Society of Pakistan (NCSP), National Cancer Control Plan, and Society of Medical Oncology Pakistan as they have plans for national cancer control programs.

\section{CONFLICT OF INTEREST}

The author declares no conflict of interest.

\section{ACKNOWLEDGEMENTS}

I would like to acknowledge Professor Sameen Siddiqi (Chair Department of Community Health Sciences, AKUH) for his encouragement and supervision to write this review report.

\section{REFERENCES}

1. Bray F, Ferlay J, Soerjomataram I, Siegel RL, Torre LA, Jemal A. Global cancer statistics 2018: GLOBOCAN estimates of incidence and mortality worldwide for 36 cancers in 185 countries. CA Cancer J Clin 2018; 68(6): 394-424.

2. Latest global cancer data: Cancer burden rises to 18.1 million new cases and 9.6 million cancer deaths in 2018. Geneva, Switzerland,: World Health Organization 2018.

3. Bray F, McCarron P, Parkin DM. The changing global patterns of female breast cancer incidence and mortality. Breast Cancer Res 2004; 6(6): 229-39.

4. Yusuf A. Cancer care in Pakistan. Jpn J Clin Oncol 2013; 43(8): 771-5.

5. Saqib MAN, Rafique I, Qureshi H, Munir MA, Bashir R, Arif BW, et al. Burden of Tobacco in Pakistan: findings from global adult tobacco survey 2014. Nicotine Tob Res 2019; 21(1): 136.

6. Prohibition of Smoking and Protection of Non-smokers Health Ordinance Ministry of National Health Services, Regulations \& Coordination; 2002.

7. Bhurgri Y, Decullier E, Bhurgri A, Nassar S, Usman A, Brennan P, et al. A case-control study of lung cancer in Karachi, Pakistan. Int J Cancer 2002; 98(6): 952-5. 
8. Bhurgri $\mathrm{Y}$, Bhurgri A, Hassan SH, Zaidi SH, Rahim A Sankaranarayanan R, et al. Cancer incidence in Karachi, Pakistan: first results from Karachi Cancer Registry. Int J Cancer 2000; 85(3): 325-9.

9. Hirabayashi $\mathrm{Y}$, Zhang $\mathrm{M}$. Comparison of time trends in breast cancer incidence (1973-2002) in Asia, from cancer incidence in five continents, Vols IV-IX. Jpn J Clin Oncol 2009; 39(6): 411-2.

10. Kumar S, Shaikh AJ, Rashid YA, Masood N, Mohammed A, Malik UZ, et al. Presenting features, treatment patterns and outcomes of patients with breast cancer in Pakistan: experience at a university hospital. Indian J Cancer 2016; 53(2): 230-4.

11. Soomro R, Faridi S, Khurshaidi N, Zahid N, Mamshad I. Age and stage of breast cancer in Pakistan: an experience at a tertiary care center. J Pak Med Assoc 2018; 68(11): 1682-5.

12. Zaheer S, Shah N, Maqbool SA, Soomro NM. Estimates of past and future time trends in age-specific breast cancer incidence among women in Karachi, Pakistan: 2004-2025. BMC Public Health 2019; 19(1): 1001

13. Qureshi MA, Mirza T, Khan S, Sikandar B, Zahid M, Aftab M et al. Cancer patterns in Karachi (all districts), Pakistan: first results (2010-2015) from a Pathology based cancer registry of the largest government-run diagnostic and reference center of Karachi. Cancer Epidemiol 2016; 44: 114-22.

14. Daniyal M, Ahmad S, Ahmad M, Asif HM, Akram M, Ur Rehman S, et al. Risk factors and epidemiology of gastric cancer in Pakistan. Asian Pac J Cancer Prev 2015; 16(12): 4821-4.

15. Idrees R, Fatima S, Abdul-Ghafar J, Raheem A, Ahmad Z. Cance prevalence in Pakistan: meta-analysis of various published studies to determine variation in cancer figures resulting from marked population heterogeneity in different parts of the country. World $J$ Surg Oncol 2018; 16(1): 129

16. Majeed FA, Azeem AR, Farhan N. Lung cancer in Pakistan, where do we stand? J Pak Med Assoc 2019; 69(3): 405-8.

17. Masood A, Masood K, Hussain M, Ali W, Riaz M, Alauddin Z, et al. Thirty years cancer incidence data for Lahore, Pakistan: trends and patterns 1984-2014. Asian Pac J Cancer Prev 2018 19(3): 709-17.

18. Bhurgri $Y$, Shaheen $Y$, Kayani N, Nazir K, Ahmed R, Usman A, et al. Incidence, trends and morphology of ovarian cancer in Karachi (1995-2002). Asian Pac J Cancer Prev 2011; 12(6): 1567-71.

19. Badar F, Mahmood S. Hospital-based cancer profile at the Shaukat Khanum Memorial Cancer Hospital and Research Centre, Lahore, pakistan. J Coll Physicians Surg Pak 2015; 25(4): 259-63.

20. Masood K, Masood A, Zafar J, Shahid A, Kamran M, Murad S, et al. Trends and analysis of cancer incidence for common male and female cancers in the population of Punjab province of Pakistan during 1984 to 2014. Asian Pac J Cancer Prev 2015; 16(13): 5297-304

21. Masood K, Zafar T, Zafar J. A statistical evaluation of common cancer trends and cancer occurrence by age group in the population of Lahore, Pakistan, 1984-2011. J Registry Manag 2014; 41(2): 85-90.

22. Muhammad Sohaib AS. PAEC Annual Cancer Registry Report 2015-2017. Nuclear Medicine \& Oncology Division, , Islamabad Pakistan Atomic Energy Comission; 2020.

23. Badar F, Mahmood S. Cancers among children and adolescents at a cancer hospital in Pakistan. J Ayub Med Coll Abbottabad 2015; 27(4): 904-10.

24. Cancer Epidemiology and Prevention. 4th ed.; Oxford University Press: New York, 2017.

25. Akhtar A, Hussain I, Talha M, Shakeel M, Faisal M, Ameen M, et al. Prevalence and diagnostic of head and neck cancer in Pakistan. Pak J Pharm Sci 2016; 29(5 Suppl): 1839-46.

26. Khan Z, Suliankatchi RA, Heise TL, Dreger S. Naswar (smokeless tobacco) use and the risk of oral cancer in Pakistan: a systematic review with meta-analysis. Nicotine Tob Res 2019; 21(1): 32-40.

27. Batool SA, Sajjad S, Malik H. Cervical cancer in Pakistan: a review. J Pak Med Assoc 2017; 67(7): 1074-7.

28. Gul S, Murad S, Javed A. Prevalence of high risk human papillomavirus in cervical dysplasia and cancer samples from twin cities in Pakistan. Int J Infect Dis 2015; 34: 14-9.
29. Rashid MU, Khan FA, Muhammad N, Loya A, Hamann U. Prevalence of PALB2 germline mutations in early-onset and familial breast/ovarian cancer patients from Pakistan. Cancer Res Treat 2019; 51(3): 992-1000.

30. Rashid MU, Muhammad N, Amin A, Loya A, Hamann U. Contribution of BRCA1 large genomic rearrangements to earlyonset and familial breast/ovarian cancer in Pakistan. Breast Cancer Res Treat 2017; 161(2): 191-201.

31. Liede A, Malik IA, Aziz Z, Rios Pd Pde L, Kwan E, Narod SA. Contribution of BRCA1 and BRCA2 mutations to breast and ovarian cancer in Pakistan. Am J Hum Genet 2002; 71(3): 595606.

32. Wilson LF, Antonsson A, Green AC, Jordan SJ, Kendall BJ, Nagle $\mathrm{CM}$, et al. How many cancer cases and deaths are potentially preventable? Estimates for Australia in 2013. Int J Cancer 2018; 142: 691-701.

33. Brown KF, Rumgay H, Dunlop C, Ryan M, Quartly F, Cox A, et al. The fraction of cancer attributable to modifiable risk factors in England, Wales, Scotland, Northern Ireland, and the United Kingdom in 2015. Br J Cancer 2018; 118(8): 1130-41.

34. Saeed S, Khan JA, Iqbal N, Irfan S, Shafique A, Awan S. Cancer and how the patients see it; prevalence and perception of risk factors: a cross-sectional survey from a tertiary care centre of Karachi, Pakistan. BMC Public Health 2019; 19(1): 360.

35. Naqvi AA, Zehra F, Ahmad R, Ahmad R, Ahmad N, Yazdani N, et al. Awareness, knowledge and attitude towards breast cancer, breast screening and early detection techniques among women in Pakistan. J Pak Med Assoc 2018; 68(4): 576-86.

36. Khan GJ, Naeem HS, Khan S, Jamshaid T, Sajid MI, Bashir I, et al. Understanding and responsiveness level about cervical cancer and its avoidance among young women of Pakistan. Asian Pac J Cancer Prev 2014; 15(12): 4877-83.

37. Arif M, Raza H, Sohail Afzal M. Alarming situation of breast cancer in women of Lahore, Pakistan from 1984 to 2018. Breast Cancer (Auckl) 2018; 12: 1178223418816093.

38. Hameed KI, Mahmood HZ, Sarfraz MD, Masood GK, Zaman S. Pathways to care for patients in Pakistan experiencing signs or symptoms of breast cancer. Breast 2019; 46: 40-7.

39. Siddiqui DE, Ashraf MS, Iftikhar S, Belgaumi AF. Predictors of treatment abandonment for patients with pediatric cancer at Indus Children Cancer Hospital, Karachi, Pakistan. Pediatr Blood Cancer 2018; 65(2): e26818.

40. Basharat S, Shaikh BT, Rashid HU, Rashid M. Health seeking behaviour, delayed presentation and its impact among oral cancer patients in Pakistan: a retrospective qualitative study. BMC Health Serv Res 2019; 19(1): 715.

41. Jamshed A, Syed AA, Shah MA, Jamshed S. Improving cancer care in Pakistan. South Asian J Cancer 2013; 2(1): 36-7.

42. Aziz Z, Iqbal J, Akram M. Effect of social class disparities on disease stage, quality of treatment and survival outcomes in breast cancer patients from developing countries. Breast J 2008; 14(4): 372-5.

43. Vaccarella S, Laversanne M, Ferlay J, Bray F. Cervical cancer in Africa, Latin America and the Caribbean and Asia: Regional inequalities and changing trends. Int J Cancer 2017; 141(10): 1997-2001.

44. Loya A, Serrano B, Rasheed F, Tous S, Hassan M, Clavero O, et al. Human papillomavirus genotype distribution in invasive cervical cancer in Pakistan. Cancers (Basel) 2016; 8(8): 72.

45. Hasan F, Mahmood Shah SM, Munaf M, Khan MR, Marsia S, Haaris SM, et al. Barriers to colorectal cancer screening in Pakistan. Cureus 2017; 9(7): e1477.

46. Yusuf A. Cancer care in Pakistan. Jpn J Clin Oncol 2013; 8(43): 771-5.

47. Majeed MH, Nadeem R, Khokhar MA, Qaisar MN. Adequacy of pain control in patients with advanced cancer in Pakistan. J Palliat Care 2019; 34(2): 126-31

48. Sutton PM. Echoes in the cancer hospital playroom: time for palliative care in Pakistan. J Palliat Med 2014; 17(6): 639.

49. Zaidi AA, Ansari TZ, Khan A. The financial burden of cancer: estimates from patients undergoing cancer care in a tertiary care hospital. Int J Equity Health 2012; 11: 60. 